18. Kristian, J., Sandage, A. R. \& Westphal, J. A. Astrophys. J. 221, 383 (1978)

19. Schmidt, M. Astrophys J. 151, 393 (1968).

20. Gunn, J. E., Lee, B. W., Lerche, I., Schramm, D. N. \& Steigman, G. Astrophys. $J$ (in the press).

1. Yahil, A., \& Beaudet, G. Astrophys. J. 206, 26 (1976).

.

23. Barnothy, J. M. \& Barnothy, M. F. Bull. Am. astr. Soc. 3, 472 (1971).

\section{Diffuse $\gamma$-ray background from Seyfert galaxies}

At least $20 \%$ of all Type I Seyfert galaxies are detectable ${ }^{1,2}$ (at the comparable Ariel 5 and Uhuru sensitivities) as X-ray sources in the $\sim 2-10 \mathrm{keV}$ band. Both the Ariel 5 and Uhuru results indicate the $\mathrm{X}$-ray luminosity function is quite steep $\left(\mathrm{d} N / \mathrm{d} L \sim L^{-2}\right)$ so that more sensitive HEAO X-ray detectors may reveal that nearly all Type I Seyfert galaxies are X-ray sources. It is suggested here that the diffuse background spectrum observed at hard X-ray and $\gamma$-ray energies can be accounted for by Seyferts, given their individual (hard) spectra.

As $4 \mathrm{U} 1410-03$ has been precisely located $^{3}$ as coincident with NGC5506, which may be a Type 2 Seyfert but morphologically resembles Cen $\mathrm{A}$, the integrated $\mathrm{X}$-ray emission from all emission line galaxies and active galaxies may exceed the $\sim 15 \%$ contribution previously estimated ${ }^{1,2}$ for the diffuse $\mathrm{X}$-ray background at $\sim 2-10 \mathrm{keV}$. If these sources typically have photon spectra flatter than $\mathrm{d} N / \mathrm{d} E \sim E^{-2,4}$, which is ${ }^{4}$ the approximate diffuse background above $\sim 20 \mathrm{keV}$, their contribution to the diffuse background spectrum at energies $E>\bar{E}_{\mathrm{c}}$ will become $\sim 100 \%$. The critical energy

$$
\bar{E}_{\mathrm{c}} \simeq\left[31.9 \int_{2}^{10} E^{-\alpha+1} \mathrm{~d} E\right]^{1 /(2.4-\alpha)} \mathrm{keV}
$$

is the energy at which the average Seyfert spectrum, $\mathrm{d} N / \mathrm{d} E=$ $A E^{-\alpha}$, crosses the diffuse background spectrum. Thus to account for all of the high energy diffuse background flux, individual Seyferts and active galaxies must typically have high energy cutoffs with $E_{\mathrm{c}} \widetilde{>} \vec{E}_{\mathrm{c}} \simeq 300 \mathrm{keV}-10 \mathrm{MeV}$ for $\alpha \sim 1.5-2$.

Some recent observations suggest this may usually be true. The hard X-ray flux from the archetypical Type $1 \mathrm{X}$-ray Seyfert, NGC4151, has now been detected (with $\alpha \sim 1.5$ ) through at least $\sim 200 \mathrm{keV}$ and possibly several MeV (refs 5, 6). As NGC 4151 is relatively close and among the brightest X-ray Seyferts, its detection is reminiscent of the detection ${ }^{7}$ of the nearby active galaxy Cen $\mathrm{A}$ with $\alpha \simeq 1.9$ at several $\mathrm{MeV}$. Increased sensitivity would confirm whether these spectra are typical for more distant members of each class of object. In fact, the Type I Seyfert $3 \mathrm{C} 120$ (with $z=0.032$ ) has also been positively identified $^{8}$ as an X-ray source and is fit by a spectral index $\alpha \simeq 1$.2-2.0. At least one much more distant $(z \simeq 0.047)$ Seyfert has now also been identified ${ }^{9}$ with $4 \mathrm{U} 0241+61$, which has ${ }^{10}$ a very hard spectrum $(\alpha \simeq 1.0)$ through $\sim 1 \mathrm{MeV}$ and then steepens (with $\alpha \simeq 3$ ) at energies through $\sim 100 \mathrm{MeV}$, if its identification with the COS-B $\gamma$-ray source ${ }^{10}$ CG135+1 is correct.

Thus in all cases where spectra have been measured for individual Seyferts and active galaxies, their spectra are both hard $(\alpha \sim 1-2)$ and extend to cutoff energies $\left(E_{\mathrm{c}} \sim 100 \mathrm{keV}-1\right.$ $\mathrm{MeV})$ large enough for their summed spectra to account for the diffuse background at energies $\widetilde{>} \bar{E}_{\mathrm{c}} \sim 100 \mathrm{keV}$. Where $\gamma$-ray spectra at $E \sim 1-100 \mathrm{MeV}$ are measured ${ }^{10,11}$ or at least limited ${ }^{12}$ (as for Cen A; C. Fichtel, personal communication) there is direct evidence for a high energy cutoff $\left(E_{\mathrm{c}} \sim 1-10 \mathrm{MeV}\right)$ or steepening with $\alpha \sim 3$. This spectral shape can be explained by Compton-synchroton models ${ }^{12}$ where the typically flat spectrum of a selfabsorbed synchrotron source in the galaxy nucleus is scattered into a self-similar spectrum in the $X$ ray- $\gamma$ ray range. The cut off is then due to the primary spectrum cut off in the infrared range where synchrotron lifetime effects are usually important, and variations (time scales $\widetilde{>} \mathrm{h}-\mathrm{d}$ ) in the hard X-ray flux correlated with changes in the radio-infrared spectrum are expected ${ }^{12}$
The diffuse background spectrum might then be expected to show a flattening at $E \sim \bar{E}_{\mathrm{c}}$, where the Seyfert contribution is $\sim 100 \%$, and a steepening at higher energies. The most recent summary ${ }^{13}$ of low energy $(\widetilde{>} 1 \mathrm{MeV}) \gamma$-ray data and high energy

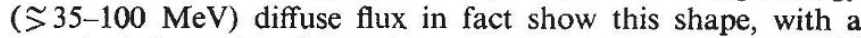
marginal flattening $(\alpha<2.4)$ at $\sim 1-10 \mathrm{MeV}$ and $\alpha \simeq 2.85_{-0.3}^{+0.5}$ at $E\ulcorner 35 \mathrm{MeV}$. As the contribution $(\sim 15 \%)$ of Seyferts in the $2-10 \mathrm{keV}$ range satisfies the limits for granularity of the background $^{2}$, so also must their dominant contribution at high energies. Finally, if all galaxies have recurrent active phases or evolve through a Seyfert-like stage, then perhaps their typically hard spectra with a range of apparent cutoffs $E_{\mathrm{c}}>E_{\mathrm{c}, \min } \simeq 20$ $\mathrm{keV}$ could account for the entire diffuse flux with $\alpha \simeq 1.4$ $\left(E \leqslant E_{\mathrm{c}, \min }\right)$ and $\alpha \simeq 2.4\left(E>E_{\mathrm{c}, \min }\right)$. However, whereas this is questionable due to uncertain evolutionary effects, the contribution now observed for (primarily) Type 1 Seyferts at least strongly suggests that Seyferts can account for all of the diffuse background at hard X-ray through $\gamma$-ray energies.

Note added in proof: Strong observational support for the Seyfert origin of the diffuse background is given by the recent announcement by Schonfelder at the Symposium on $\gamma$-ray Spectroscopy in Astrophysics (Goddard Space Flight Center, 28 April 1978) that $\gamma$ rays have been detected from NGC4151. The spectrum seems to extend the power law from the hard $\mathrm{X}$ ray range to a treak at about $3 \mathrm{MeV}$, and is thus consistent with our prediction for the individual sources comprising the diffuse spectrum.

\section{Jonathan E. Grindlay}

Harvard-Smithsonian Center for Astrophysics,

60 Garden Street, Cambridge, Massachusetts 02138

Received 3 January; accepted 28 February 1978.

1. Elvis, M. et al. Mon, Not, $R$ astr. Soc (submitted).

. Tananbaum, H., Peters, G., Forman, W., Giacconi, R. \& Jones, C. A strophys. J. (submitted).

3. Ricker, G. et al. Astrophys. J. Lett. (submitted).

4. Schwartz, D. \& Gursky, H. in X-ray A stronomy (eds Giacconi, R. \& Gursky, H.) 359 (Reidel, Dordrecht, 1974).

5. Baity, W., Jones, T., Wheaton, W. \& Peterson, L. Astrophys. J. Lett. 199, L5-L8 (1975).

. Di Cocco, G, et al. Nature 270, 319-320 (1977)

7. Hall, R., Meegan, C., Walraven, G., Djuth, F. \& Haymes, R. Astrophys. J. 210, 631-641 (1976)

. Schnopper, H. W., Epstein, A., Delvaille, J. P., Tucker, W., Doxsey, R. \& Jernigan, G. Astrophys. J. Lett. 215, L7-L.11 (1977).

9. Apparao, K. et al. IAU Circ. No. 3150 (1977).

10. Maraschi, L. et al. Nature 272, 679-681 (1978).

11. Hermsen, W. et al. Nature 269, 494-495 (1977).

2. Grindlay, J. E. Astrophys. J. 199, 49-53 (1975).

3. Fichtel, C., Simpson, G. \& Thompson, D. Astrophys. J. (submitted).

\section{Coherently overturned flaps surrounding craters}

MANY large impact and explosion craters exhibit coherently overturned flaps in the form of ejecta blankets ${ }^{1-7}$ in which the pre-existing stratigraphy of the cratered ground is preserved in detail, but inverted. Sequential ejection of material into ballistic trajectories could produce a coarsely graded inversion of the material, but would not retain detailed stratigraphic coherence. The field evidence from fully excavated craters $^{3-7}$ shows that even totally incompetent strata composed of free flowing sand may be traced continuously through a hinge region below the crater rim outwards to at least three times the crater rim radius. The strata retain their integrity and relative position despite being reduced by stretching from an original thickness of some tens of centimetres to less than a centimetre at the extreme limit of coherent overturning. Figures 1 and 2, a photograph and a stratigraphic plot ${ }^{3}$ of the hinge region of the Prairie Flat crater at the Defence Research Establishment, Suffield, illustrate the phenomenon. Similar examples are given in the reports of the excavation ${ }^{3-4}$. The effect was 